\title{
Characterisation of a multi-channel multiplexed EMG recording system: towards realising variable electrode configurations
}

\author{
$1^{\text {st }}$ Kylie de Jager $2^{\text {nd }}$ Michael Mentink $3^{\text {rd }}$ Henry Lancashire $4^{\text {th }}$ Yazan Al-Ajam $5^{\text {th }}$ Stephen Taylor $6^{\text {th }}$ Anne Vanhoestenberghe \\ Aspire CREATe, UCL \\ London, UK \\ UREST Ltd. \\ $I D G, U C L$ \\ $B M E, U C L \quad$ Aspire CREATe, UCL \\ Basingstoke, UK \\ London, UK \\ London, UK \\ London, UK \\ Aspire CREATe, UCL \\ michael@urest.uk \\ h.lancashire@ucl.ac.uk ajam@doctors.org.uk stephen.taylor@ucl.ac.uk \\ London, UK \\ a.vanhoest@ucl.ac.uk
}

\begin{abstract}
An implantable EMG amplifier with a novel multiplexed frontend for in vivo selection of optimal electrode configurations, was designed using commercially available components. The multiplexers are advantageous as optimal electrode configuration are not known before implantation. The system has 6 ADC recording channels (ADS1298 biopotential amplifier, $2 \mathrm{kHz}$ sampling frequency, 16-bit resolution), and three $8 \times 8$ multiplexer arrays (ADG2188), 2 channels per MUX. The system was characterised by measuring input impedance $(5.8 \mathrm{MOhm})$ and frequency response (CMRR 49.0 dB; SNR 51.4 dB). EMG recordings from implanted epimysial electrodes showed lower signal quality (13.5 dB) compared with a commercial EMG recorder $(19.5 \mathrm{~dB})$, nonetheless the signals appeared suitable for myoelectric control applications. An implantable version of the EMG recorder, housed within a hermetically sealed ceramic package, should improve signal quality.

Index Terms-Biopotential recordings, electromyography, electrode array, bipolar, tripolar, monopolar, implantable electronics.
\end{abstract}

\section{INTRODUCTION}

Socket and strap fitted prostheses are known to cause skin irritation and sores. One possible solution to avoid such complications is to load the bone instead of the skin, through direct skeletal fixation via a permanently inserted bone anchor with soft tissue flange [1]. As a first step towards achieving osseointegrated myoelectrically-controlled prostheses, external EMG recording equipment can be used in combination with a bone anchor conduit [2], through which EMG signals from implanted electrodes can be passed [3].

The quality, or signal-to-noise ratio (SNR), of such EMG recordings is dependent on the electrode configuration to optimise the electrode pick-up area [4; 5]. Al-Ajam et al. [6] proposed incorporating multiplexer functionality in an EMG recording device, which in combination with bone anchor feedthrough, will provide versatility of electrode configurations. This will enable in vivo reconfigurations, increasing the SNR as the optimal electrode configuration changes after implantation.

The design and development of an EMG recorder with a multiplexer frontend, referred to as the CAPITel (Control of Active Prostheses using Implantable Telemetry) system was previously presented in [7]. This paper expands on the earlier work with a thorough system characterisation, in vivo recordings and comparison with a commercial system. The prototype presented was tested as an external device, however

Thanks to the Restoration \& Function Trust (RAFT, UK), the Therapeutic Acceleration Support (TAS) Fund and the Wellcome Trust [grant number $106574 / Z / 14 / Z]$ for financial support. it is designed to be housed in an implantable, hermetically sealed, ceramic package, for use in animal models for further research before implementing the final circuit as an ASIC.

\section{METHOD}

\section{A. System design and performance evaluation}

Figure 1 shows the concept of the CAPITel system (specifications listed in Table II). Multiplexers (MUX), in the frontend of the EMG recorder, allow a variety of electrode configurations from 6-pole electrode arrays, shared between 2 recording channels of a multi-channel analog-to-digital converter (ADC) with adjustable gain. A bandpass filter (BPF), between the MUX and ADC, limits the recorded EMG bandwidth. The digital output is communicated via SPI directly to a microcontroller and via Bluetooth to a laptop for recording. The micro-controller, in combination with a LabVIEW interface,

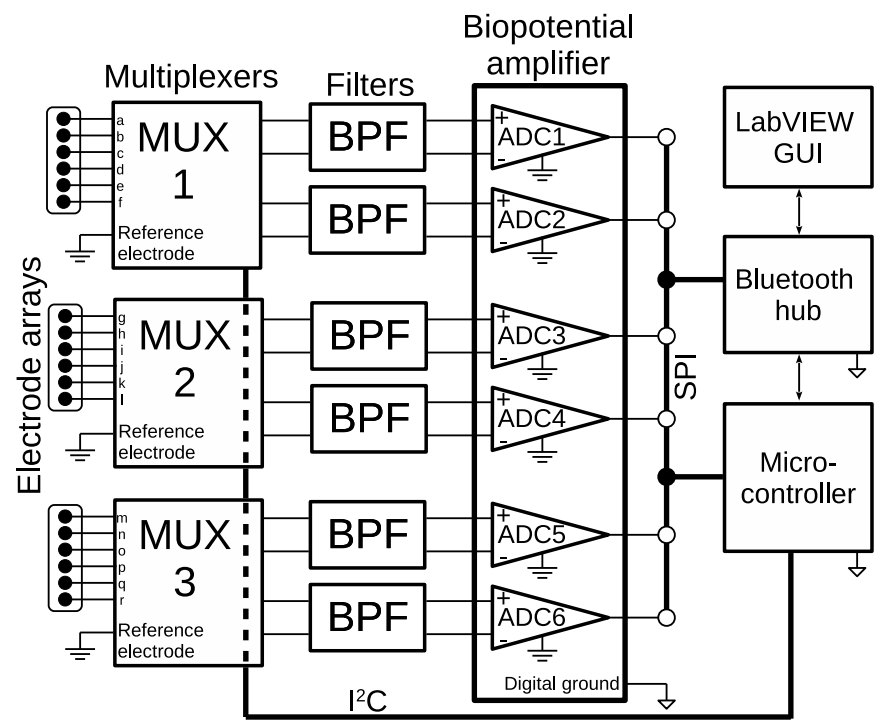

Fig. 1: Block diagram of the CAPITel system showing the frontend MUXs, analogue BPFs and a 6 channel ADC.

TABLE I: RANDOMISED BIPOLAR CONNECTIONS DURING CHARACTERISATION TESTS.

\begin{tabular}{l|llllll}
\hline Input terminal & $\mathbf{1}$ & $\mathbf{2}$ & $\mathbf{3}$ & $\mathbf{4}$ & $\mathbf{5}$ & $\mathbf{6}$ \\
\hline Non-inverting (+) & $\mathrm{e}$ & $\mathrm{a}$ & $\mathrm{j}$ & $\mathrm{k}$ & $\mathrm{n}$ & $\mathrm{p}$ \\
Inverting (-) & $\mathrm{c}$ & $\mathrm{d}$ & $\mathrm{h}$ & $\mathrm{g}$ & $\mathrm{m}$ & $\mathrm{q}$ \\
\hline
\end{tabular}




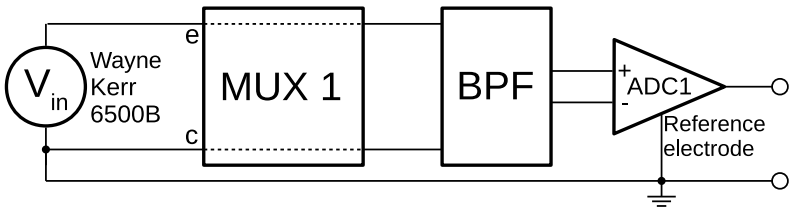

(a) Impedance analyser setup for $\mathrm{ADC}_{1}$.

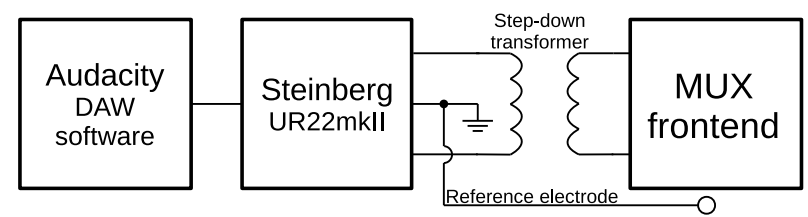

(b) Balanced bipolar sine-wave test-bench: Audacity digital audio work station; UR22mkII Steinberg audio interface; step-down transformer.

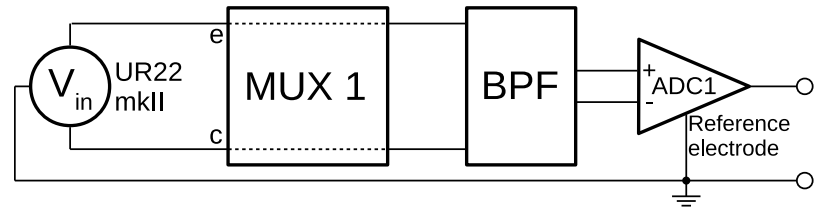

(c) Differential mode setup for $\mathrm{ADC}_{1}$.

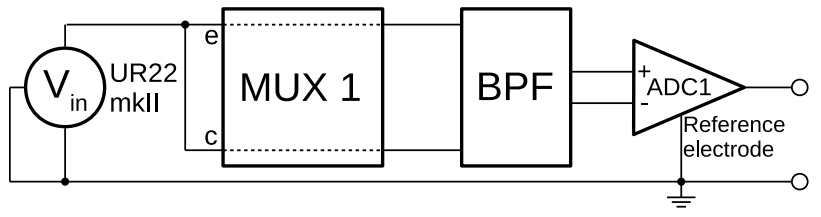

(d) Common mode setup for $\mathrm{ADC}_{1}$.

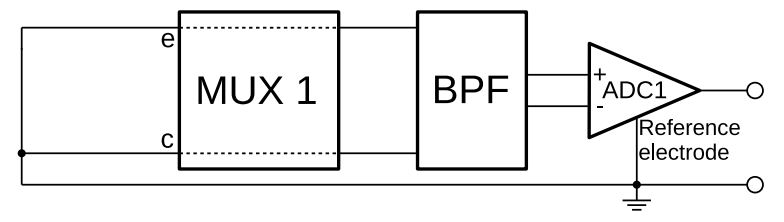

(e) Baseline noise setup for $\mathrm{ADC}_{1}$.

Fig. 2: (a) Test-bench used for system characterisation. (b-d) Internal connections realised with MUX (dashed lines) and external connections for characterising the system impedance, frequency response and SNR of $\mathrm{ADC}_{1}$. Similar setups were used for each ADC channel bipole connection (Table I).

allows the user to control the ADC gain and define the switch state of the 3 MUXes.

Characterisation tests provide a framework to evaluate the performance of the system. Due to the many electrode configurations realisable with the MUX frontend, each recording channel was characterised for a single, randomly selected, bipolar configuration (Table I).

\section{B. Input impedance}

An EMG recording system requires an input impedance greater than $1 \mathrm{M} \Omega$ to account for the electrode impedance; Al-Ajam et al. [6] measured epimysial electrode impedances of $2.3 \mathrm{k} \Omega$. A Wayne Kerr $6500 \mathrm{~B}$ impedance analyzer $(20 \mathrm{~Hz}$ - $100 \mathrm{kHz}$ frequency range; $1 \mathrm{~mA}$ drive current) was used to measure input impedance (Fig. 2a).

\section{Frequency response and signal-to-noise ratio (SNR)}

Figure $2 \mathrm{~b}$ shows the test-bench used to generate a balanced bipolar sine-wave test signal (frequency range: $1 \mathrm{~Hz}-10 \mathrm{kHz}$ ).
This signal was used as both a differential mode (DM) and a common mode (CM) source (Fig. $2 \mathrm{c}$ and $2 \mathrm{~d}$ respectively). The peak-to-peak DM input voltage amplitude $\left(10.6 \mathrm{mV}_{p p}\right)$ approximates the expected EMG amplitude, while a larger $\mathrm{CM}$ input voltage $\left(337.5 \mathrm{mV}_{p p}\right)$ ensured an output signal was detectable to calculate the attenuation. The internal gain of the ADC channels was set to $6 \times$ for all measurements.

For SNR, the baseline noise RMS voltage was measured with the input terminals shorted to the reference potential (Fig. 2e). The RMS voltage measured for the DM source (10.6 $\mathrm{mV}_{p p}$ input voltage) was used as the signal RMS voltage.

$$
\mathrm{SNR}=20 \cdot \log _{10}\left(\frac{v_{S, r m s}}{v_{N, r m s}}\right)
$$

\section{Power consumption}

The power consumption of the system was found by measuring the voltage over a $10 \Omega \pm 1 \%$ resistor, connected in series with the supply line.

\section{RESULTS}

\section{A. Circuit design}

Figure 3 shows the CAPITel circuit, see Table II for design criteria. The core is an ADS1298 ADC biopotential amplifier ( 6 channels, 16-bit resolution, $2 \mathrm{kHz}$ sampling rate, $1 \times$ to $12 \times$ variable gain, $500 \mathrm{~Hz}$ bandwidth). It has a small footprint $(8 \times 8$ $\mathrm{mm})$ and low power consumption $(6 \mathrm{~mW})$, both necessary for an implantable device. Further benefits of the IC are a low input-referred noise $\left(12.4 \mu \mathrm{V}_{r m s}\right)$ and wide supply voltage range (digital $5.25 \mathrm{~V}$; analogue $3.6 \mathrm{~V}$ ).

Three low current ADG2188 multiplexers were used (2 ADC channels per MUX, $8 \times 8$ array, $1 \mu$ A quiescent supply). Although the ADG2188 has a large footprint $\left(25 \mathrm{~mm}^{2}\right)$ in comparison to switch-type MUX $\left(3-8 \mathrm{~mm}^{2}\right)$, it saves space by only requiring $2 \mathrm{I}^{2} \mathrm{C}$ control lines for all $3 \mathrm{MUX}$, and the array functionality allows any possible combination of a 6-pole electrode array to be realised.

An analogue bandpass filter $(30-800 \mathrm{~Hz})$ was incorporated to maximise the SNR. The balanced differential topology minimises the number of components ( 9 per ADC channel), hence the surface area required on the PCB. Simulations of the filter show an attenuation of $-2 \mathrm{~dB}$ in the passband.

A MSP430FR2433 micro-controller (126 $\mu \mathrm{A} / \mathrm{MHz}$; SPI and $\mathrm{I}^{2} \mathrm{C}$ interface; $4 \times 4 \mathrm{~mm}$ ) communicates with the $\mathrm{ADC}$ and the MUX. An external Bluetooth hub (not shown in Fig. 3) transmits the ADC SPI output to a laptop.

\section{B. Input impedance}

Figure 4 shows the measured input impedance (amplitude and phase). $Z_{\text {mean }}$ is the average of 12 impedance measurements between the electrode connection pairs (Table I), where one of the pair was connected to ground. This shows frontend impedance to ground (including MUX, BPF and ADC).

$Z_{\text {short-circuit }}$, a single impedance measurement between two electrode inputs short-circuited through the MUX, shows the impedance of the MUX.

\section{Frequency response and signal-to-noise ratio (SNR)}

Figure 5 shows a Bode plot of the differential and common mode gain ( $A_{D M}$ and $A_{C M}$ respectively); phase plots have not been included as we could not determine the phase from the digital output of the CAPITel system. The Common Mode 


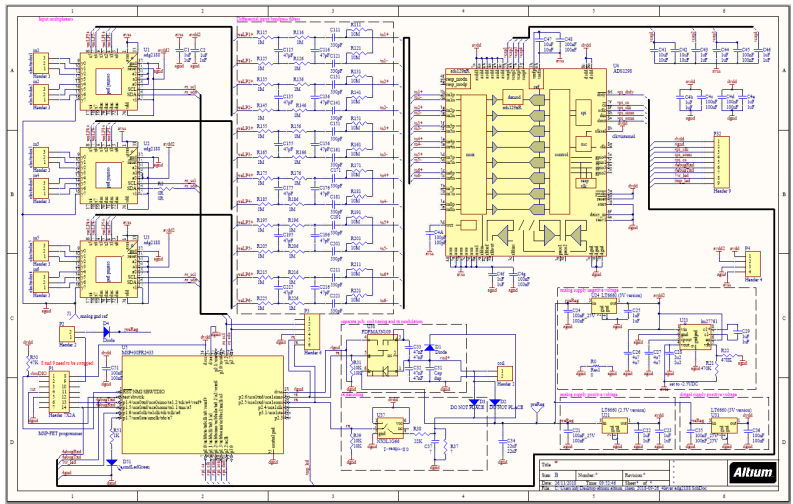

(a) Schematic

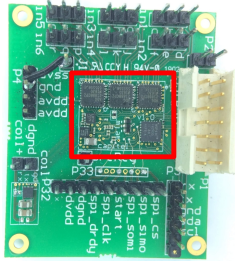

(b) Top layout.

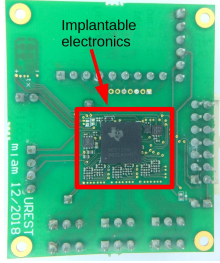

(c) Bottom layout.
Fig. 3: The double sided PCB layout is contained within a $19.33 \times 14.37$ $\mathrm{mm}$ area (red outline), to be packaged in an implantable, hermetically sealed ceramic housing, in later revisions.

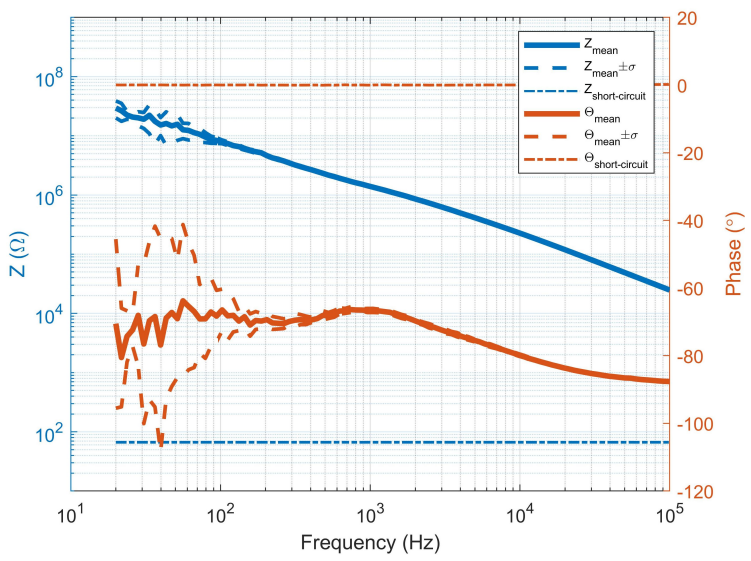

Fig. 4: Input impedance and phase measured with a Wayne Kerr 6500B impedance analyser.

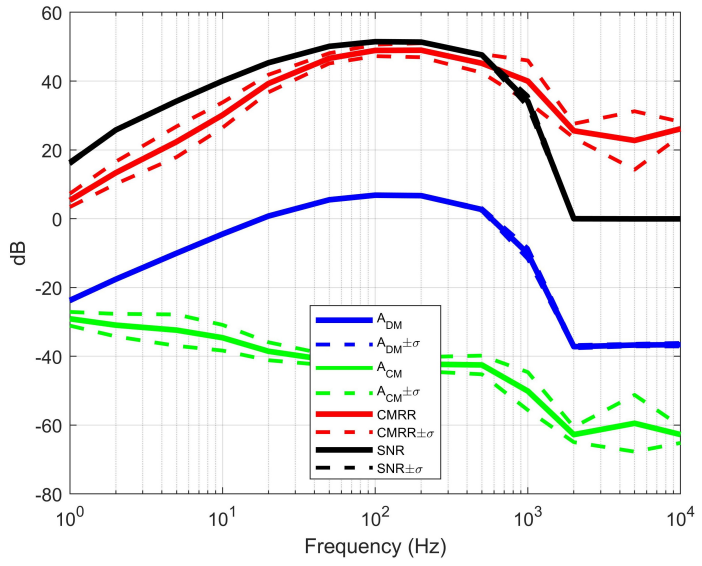

Fig. 5: Mean and standard deviation $(\sigma)$ DM, CM, CMRR and SNR across all six ADC channels. DM, CM and CMRR use $V_{p p}$ values, SNR uses $V_{r m s}$ values. Sine-wave test signal amplitudes: $\mathrm{DM} 10.6 \mathrm{mV}_{p p}$; CM $337.5 \mathrm{mV}_{p p}$.
Rejection Ratio (CMRR) and the SNR are included in the same figure. All four traces are mean values measured with the 6 electrode connection pairs given in Table I. All 6 ADC channels had a gain of $6 \times$ during data capture. For the SNR measurements, the DC offset was removed by subtracting the mean from the output voltage, before calculating $v_{r m s}$ values.

\section{Power consumption}

When powering the system with a $5.5 \mathrm{~V}$ supply, $8 \mathrm{~mA}$ continuous current was measured when idle, increasing to 11.2 $\mathrm{mA}$ during data transmission, giving a power consumption range of $44 \mathrm{~mW}$ to $61.6 \mathrm{~mW}$.

\section{E. System performance}

Table II presents specifications and experimental results.

\section{F. In vivo EMG recordings}

Two bone-anchored devices were implanted trans-tibially in an ovine model [6] to provide a hard-wired interface for two epimysial electrode arrays sutured onto the peroneus tertius muscle (5-electrode array) and gastrocnemius (2-electrode array). The CAPITel system realised monopolar, bipolar and tripolar electrode configurations with the 5-electrode array. The same configurations were also realised via a purpose-built switch arrangement [8] connected to a BIOPAC MP150 with EMG100C bioamplifiers (BIOPAC Systems UK, $100 \mathrm{~Hz}$ to $500 \mathrm{~Hz}$ bandpass, $1000 \times$ gain, $2 \mathrm{kS} / \mathrm{s}$ ). EMG was recorded with both systems during walking. The CAPITel recordings were post-processed through a second order Butterworth bandpass filter $(10 \mathrm{~Hz}$ to $500 \mathrm{~Hz})$ for direct comparison with the BIOPAC results.

Figure 6 ( $a$ and $b)$ shows the frequency spectrum (FFT) of the EMG (c and d) captured with both EMG recording systems for a bipolar electrode configuration. The signal in the shaded intervals is greater than 1.5 times standard deviation above baseline, considered meaningful EMG activity. Nonshaded intervals were considered to be noise. Equation 1 was used to calculate the SNR for the CAPITel system (13.5 dB) and the BIOPAC system (19.5 dB).

\section{DISCUSSION}

The CAPITel system is unique in that it adds multiplexers to an EMG recorder frontend enabling the in vivo selection of electrode configurations. This is advantageous for implanted systems, as: patient-specific optimal configurations, influenced by the exact placement of electrodes on the target muscles, can

TABLE II: DESIGN SPECIFICATIONS AND EXPERIMENTAL OUTCOME FOR THE CAPITEL SYSTEM.

\begin{tabular}{l|l|l}
\hline Parameter & $\begin{array}{l}\text { Design } \\
\text { Criteria }\end{array}$ & Experimental Outcome \\
\hline $\begin{array}{l}\text { Recording channels } \\
\text { Sampling frequency }\end{array}$ & 6 & $\begin{array}{l}6 \mathrm{ADCs}(\mathrm{ADS} 1298) \\
2 \mathrm{kHz}\end{array}$ \\
$\begin{array}{l}\text { Input impedance } \\
-Z_{\text {mean }}\end{array}$ & $>1 \mathrm{MHz}$ & $5.8 \pm 0.3 \mathrm{M} \Omega\left(-70.4^{\circ} \pm 3.3^{\circ}\right)^{a b}$ \\
$-Z_{\text {short-circuit }}$ & $30-800 \mathrm{~Hz}$ & $20-6 \Omega\left(-0.1^{\circ}\right)^{a b}$ \\
Bandwidth & $0-40 \mathrm{~dB}$ & $6.8 \pm 0.1 \mathrm{~dB}^{a}$ \\
Gain (passband) & $40 \mathrm{~dB}$ & $49.0 \pm 1.9 \mathrm{~dB}^{a}$ \\
CMRR (passband) & $>30 \mathrm{~dB}$ & $51.4 \pm 0.2 \mathrm{~dB}^{a}$ \\
SNR & $<100 \mathrm{~mW}$ & $44-61.6 \mathrm{~mW}$ \\
Power consumption & $<10$
\end{tabular}

$\bar{a}$ Measured at $155 \mathrm{~Hz}$; centre frequency of design criteria bandwidth (chosen to prevent contamination from $50 \mathrm{~Hz}$ harmonics). ${ }^{b}$ Amplitude (phase). 


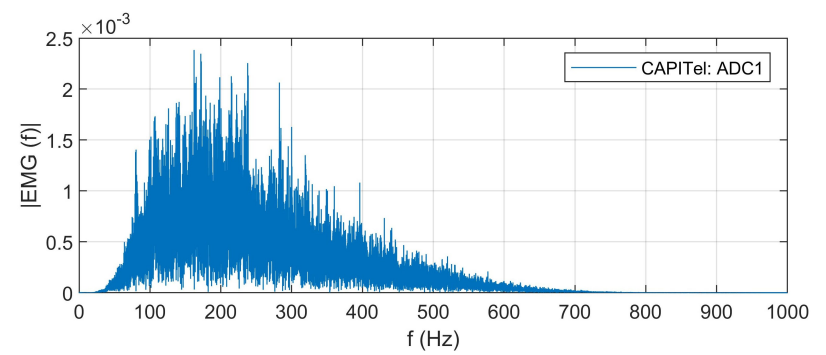

(a) Capitel FFT

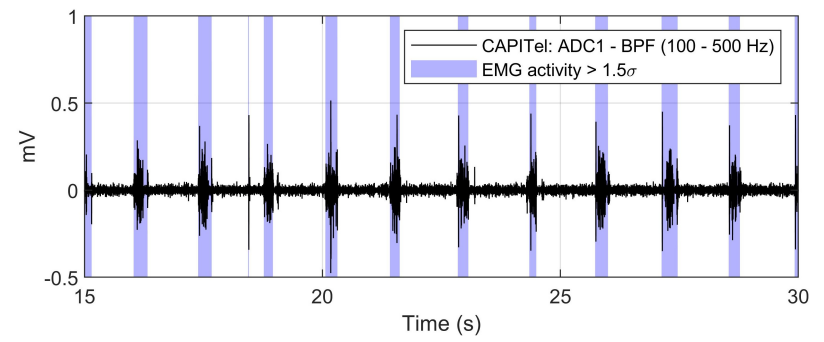

(c) Capitel EMG.

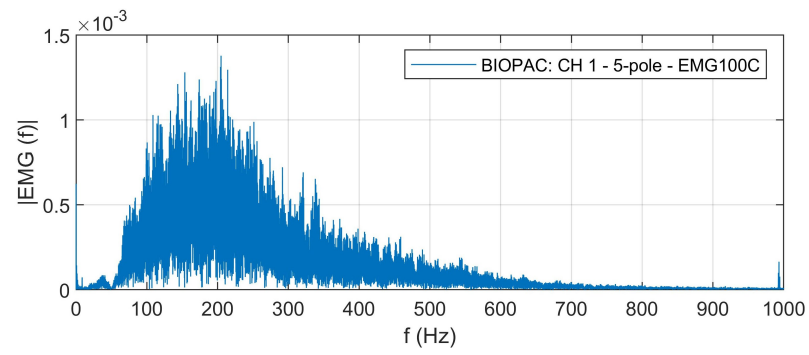

(b) Biopac FFT.

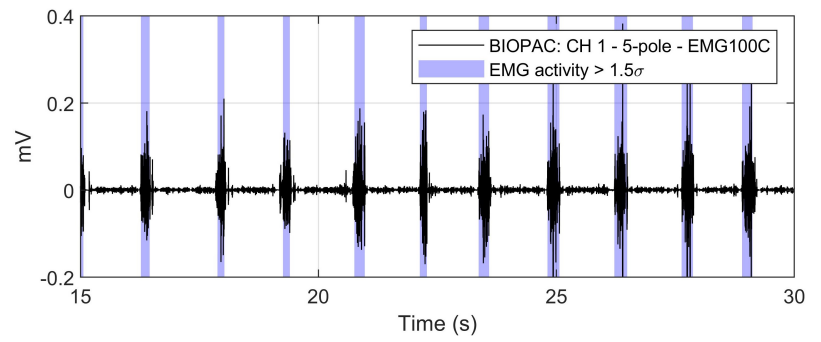

(d) Biopac EMG.

Fig. 6: Comparison of the CAPITel system (a and c) against a commercial BIOPAC EMG recorder (b and d). The CAPITel results were passed through a $100 \mathrm{~Hz}$ to $500 \mathrm{~Hz}$ bandpass Butterworth filter $(n=2)$ during post-processing to remove DC offset and for direct comparison with the BIOPAC data.

be identified post surgery; and drift in electrode impedance over time can be accounted for without the need for revision surgery.

This proof-of-concept design (fully implantable once encapsulated) was achieved using standard PCB and commercially available components without expensive and time consuming ASIC development.

The presence of the additional frontend components decreases the system performance below that of the ADS1298 (500 M $\Omega$ input impedance; $75 \mathrm{~dB}$ SNR for a $10 \mathrm{mV}$ sine-wave test signal; $108 \mathrm{~dB}$ CMRR at $155 \mathrm{~Hz}, 5.6 \mathrm{~V}$ input signal); CAPITel (input impedance dominated by BPF impedance, $10 \mathrm{M} \Omega$; SNR has $20 \mathrm{~dB}$ loss in frontend, MUX and BPF). Some differences are also due to the characterisation test conditions as $A_{C M}$ was measured with an input signal of 337.5 $\mathrm{m} V_{p p}$. Such a payoff is to be expected, but should not deter researchers from utilising encapsulated PCBs when developing implantable prototypes for animal studies as the characterised system still meets the design criteria.

At present CAPITel does not perform as well as the commercial BIOPAC system (section III-F) even after bandpass filtering during post-processing. Nonetheless the signal quality was sufficient for the intended application, namely to identify EMG activity as necessary for myoelectric control.

Further developing CAPITel to realise an implantable EMG recorder will reduce the complexity of implant-prosthesis connection, in this instance reducing from 19 lines $(3 \times 6$ electrodes +1 reference) to 6 lines ( 3 SPI + UART + power + ground). This hard-wired link to power the implant and to facilitate bidirectional serial communications, is feasible in combination with a bone anchor conduit. Applications for which the bone anchor is not suitable, will operate with a wireless link.

\section{CONCLusions}

An EMG recorder with multiplexing functionality at its frontend was presented. This will allow for in vivo selec- tion and optimisation of electrode configurations. The performance of the system was characterised by measuring input impedance, frequency response and SNR. The system performed within the required specifications. EMG recordings had lower SNR values compared with measurements captured with a commercial BIOPAC system. Nonetheless, the signal quality was sufficient to detect the presence of EMG activity and will be suitable for myoelectric control of prostheses.

\section{REFERENCES}

[1] C. J. Pendegrass, A. E. Goodship, and G. W. Blunn, "Development of a soft tissue seal around bone-anchored transcutaneous amputation prostheses," Biomaterials, vol. 27, no. 23, pp. 41834191, 2006.

[2] F. Tjulkins, H. Lancashire, K. de Jager, and A. Vanhoestenberghe, "Transcutaneous Feedthrough Enabled Bone Anchor for Attachment and Control of a Smart Upper Limb Prosthesis," in BioMedEng 2018, 2018, p. 396.

[3] M. Ortiz-Catalan, B. Håkansson, and R. Brånemark, "An osseointegrated human-machine gateway for long-term sensory feedback and motor control of artificial limbs." Science translational medicine, vol. 6, no. 257, p. 257re6, 2014.

[4] T. J. Koh and M. D. Grabiner, "Evaluation of methods to minimize cross talk in surface electromyography," Journal of Biomechanics, vol. 26, pp. 151-157, 1993.

[5] J. van Vugt and J. van Dijk, "A convenient method to reduce crosstalk in surface EMG," Clinical Neurophysiology, vol. 112, no. 4, pp. 583-592, 2001.

[6] Y. Al-Ajam, H. Lancashire, C. Pendegrass, N. Kang, R. P. Dowling, S. J. G. Taylor, and G. Blunn, "The Use of a BoneAnchored Device as a Hard-Wired Conduit for Transmitting EMG Signals From Implanted Muscle Electrodes," IEEE Transactions on Biomedical Engineering, vol. 60, no. 6, pp. 1654 1659, 2013.

[7] K. de Jager, M. Mentink, F. Tjulkins, H. Lancashire, S. Taylor, and A. Vanhoestenberghe, "A multi-channel multiplexed EMG recording system to study electrode array configuration," in BioMedEng 2018, London, sep 2018, p. 367.

[8] H. Lancashire, K. de Jager, F. Tjulkins, and A. Vanhoestenberghe, "Electrode configuration selection for electromyographic prostheses," in BioMedEng 2018, London, sep 2018, p. 397. 